Institute of Public Health, Faculty of Health Sciences, University of

Copenhagen, Denmark

$\mathrm{J} C$ Norregaard

$\mathrm{T} F$ Andersen

Department of Ophthalmology, Hvidovre University

Hospital, Copenhagen,

Denmark

J C Norregaard

P Bernth-Petersen

Health Services

Research Unit, Institut

Municipal

d'Investigació Mèdica,

Barcelona, Spain

J Alonso

M Espallargues

Department of

Community Health

Sciences, Faculty of

Medicine, University

of Manitoba,

Winnipeg, Canada

E Dunn

C Black

Health Sciences Clinical Research

Centre, Winnipeg,

Canada

E Dunn

Manitoba Centre for Health Policy and

Evaluation, University of Manitoba,

Winnipeg, Canada

C Black

Department of Ophthalmology, Faculty of Medicine,

University of

Manitoba, Winnipeg,

Canada

L Bellan

Department of Health Policy and

Management, Johns

Hopkins University,

Baltimore, USA

G F Anderson

Correspondence to: Dr Jens Christian

Norregaard, Institute of

Public Health, University of

Copenhagen, Blegdamsvej 3 ,

2200 Copenhagen,

Denmark.

Accepted for publication 11 March 1998

\title{
Variation in indications for cataract surgery in the United States, Denmark, Canada, and Spain: results from the International Cataract Surgery Outcomes Study
}

Jens Christian Norregaard, Peter Bernth-Petersen, Jordi Alonso, Elaine Dunn, Charlyn Black, Tavs Folmer Andersen, Mireia Espallargues, Lorne Bellan, Gerard F Anderson

\section{Abstract}

Backgroundlaims-International comparisons of clinical practice may help in assessing the magnitude and possible causes of variation in cross national healthcare utilisation. With this aim, the indications for cataract surgery in the United States, Denmark, the province of Manitoba (Canada), and the city of Barcelona (Spain) were compared.

Methods-In a prospective multicentre study, patients scheduled for first eye cataract surgery and aged 50 years or older were enrolled consecutively. From the United States 766 patients were enrolled; from Denmark 291; from Manitoba 152; and from Barcelona 200. Indication for surgery was measured as preoperative visual status of patients enlisted for cataract surgery. Main variables were preoperative visual acuity in operative eye, the VF-14 score (an index of functional impairment in patients with cataract) and ocular comorbidity.

Results-Mean visual acuity were 0.23 (USA), 0.17 (Denmark), 0.15 (Manitoba), and 0.07 (Barcelona) $(\mathbf{p}<\mathbf{0 . 0 0 1})$. When restricting the sample to eyes with normal retina and macula, no significant difference between United States and Denmark was observed $(p>0.05)$. Mean VF-14 scores were 76 (USA), 76 (Denmark), 71 (Manitoba), and 64 (Barcelona) $(\mathrm{p}<0.001)$.

Conclusion-Similar indications for cataract surgery were found in the United States and Denmark. Significantly more restricted indications were observed in Manitoba and Barcelona. Possible explanations for the results are discussed, including differences in sociodemographic characteristics, access to care, surgeons' willingness to operate, and patient demand.

(Br f Ophthalmol 1998;82:1107-1111)
Several studies have shown variation in rate of cataract extraction among geographic regions within a single country. ${ }^{1-3}$ A study of regional variation for cataract extractions in the United States, for example, showed that the annual rate of cataract surgery varied from 3.8 to 41.2 per 1000 Medicare beneficiaries in different geographic areas. ${ }^{3}$ The study showed that multiple factors were associated with variation in the rates of surgery, including age and sex of patients, latitude and higher concentration of optometrists. However, many studies of regional variation are unable to assess the association of clinical characteristics of patients with practice variation, because they are usually based upon administrative data, which do not contain relevant clinical information. This is a limitation of such studies since there may be alternative explanations for practice pattern variation. It may be, for example, that a high rate of surgery could reflect liberal indications for surgery, while similarly a low rate could suggest access problems. To examine these hypotheses, clinical information on visual status is needed.

The study is part of the International Cataract Surgery Outcomes Study. The objective of this international comparative research project is to compare current cataract management, outcomes of surgery, and quality of care in four international sites. Data for the study were collected in the United States, Denmark, province of Manitoba (Canada), and city of Barcelona (Spain). The design of the international research programme is based on methods developed by the US National Cataract Surgery Outcomes Study. ${ }^{4}$

In the present study, we compared the visual status of patients undergoing cataract surgery in different healthcare settings in North America and Europe. The aim was to assess the magnitude of variation in indications or threshold for cataract surgery and to obtain a better understanding of possible causes of variation in cross national healthcare utilisation. 


\section{Materials and methods}

DESIGN

Data were collected at multiple clinical practices within each site. At each clinical practice location, consecutive samples of patients recommended for cataract surgery were obtained. In the United States, three cities were selected as recruitment areas based on the annual rate of cataract surgery performed on Medicare beneficiaries. ${ }^{4}$ These cities were Columbus, Ohio (low rate), St Louis, Missouri (medium rate), and Houston, Texas (high rate). Ophthalmologists at each site were categorised into strata based on annual volume of cataract surgery $(0-50,51-200,201-399$, and $>400$ surgeries per year). A stratified random sample of 75 ophthalmologists was recruited from the three strata that contained those with an annual volume greater than 50 surgeries per year. The ophthalmologists were asked to refer consecutive patients who were scheduled to undergo cataract surgery and fulfilled the criteria for inclusion. Patients were recruited consecutively from 15 July 1991 to 15 December 1991 or until 14 patients had been enrolled from each practice, whichever came first.

In Denmark, cataract surgery was performed at 17 ophthalmological departments in public hospitals, all of which agreed to participate in the study. At the time of the data collection, no more than $15 \%$ of the total volume of cataract surgery was performed in private surgical clinics. ${ }^{5}$ These clinics did not participate in the study. In the Danish public healthcare system, a patient with cataract is referred by a general ophthalmologist who has a "gatekeeper" function. The referred patient is placed on a waiting list for examination by a hospital surgeon. Beginning in September 1992, patients were enrolled consecutively for this study based on referral notes submitted to the hospitals by general ophthalmologists. Recruitment continued until the required number of patients had been enrolled. The number of enrollees per hospital department depended on the surgical volume of the department (range 7-26, mean 17 patients). The last patient was enrolled in March 1993.

The Canadian study was conducted in the province of Manitoba. Of 18 ophthalmologists performing surgery in the province, $12(67 \%)$ agreed to participate in the study. The ophthalmologists were asked to refer eligible patients consecutively as they were enlisted for surgery. The recruitment began on 1 September 1992 and continued until 28 February 1993 or until an agreed number of patients from each office was obtained, whichever came first (range 1-24, mean 13 patients).

In the city of Barcelona, Spain, approximately $40 \%$ of the cataract surgery is done in the private sector and $60 \%$ in the public sector. From the private sector, 12 ophthalmologists were randomly selected and six agreed to participate. Each ophthalmologist was asked to recruit up to 15 consecutive patients, depending on their surgical volume. From the public sector, four out of eight departments providing cataract surgery care in the city were selected after stratification based on volume of cataract surgery, average case severity, and financial arrangements of each hospital. Patients were enrolled from September 1993 to March 1994.

Patients were eligible for inclusion in the study if they were scheduled for first eye cataract surgery and were 50 years of age or older. Patients were excluded if they had previously undergone cataract surgery or if the planned cataract surgery was a combined procedure involving glaucoma, corneal, or vitreoretinal surgery. Further exclusions were made if patients were not living within specified recruitment areas, did not speak the primary language of the area, were deaf or confused, or did not have access to a telephone.

After consent was obtained, patients were contacted for an in depth telephone interview. A face to face interview was scheduled if the patient had difficulties completing an interview by telephone. The baseline patient interview collected basic demographic information, information on visual functional impairment (VF-14 score), and general health status. The VF-14 is an index of functional impairment in patients with cataract. ${ }^{4}$ Based on the ability to perform 14 daily living activities, a score is calculated ranges from 0 (maximum impairment) to 100 (no impairment). The US, Canadian, Danish, and Spanish versions of this instrument have been shown to be reliable, valid, and responsive to clinical changes following cataract surgery. ${ }^{467}$ General health status was assessed by application of the Sickness Impact Profile (SIP) ${ }^{8}$ This questionnaire is a valid and reliable measure of self reported general health status. ${ }^{8}{ }^{9}$ The SIP score ranges from 0 (indicating absence of dysfunction) to 100 (indicating the maximum level of dysfunction). A translation/backtranslation technique ${ }^{10}$ was used to develop a Danish version of the SIP. A previously adapted and validated Spanish version of the SIP was used for the Barcelona site. $^{11}$

A full preoperative medical history and ophthalmological examination of each patient was obtained including refraction, slit lamp, and fundus examination. Best corrected visual acuity was obtained on a Snellen chart. The results were reported in structured data sheets by the patients' ophthalmologists at the point in time when the decision to perform surgery was made.

The patient interview was developed by the international team based on an original US questionnaire. A translation/back translation technique was used to minimise translation bias. $^{10}$ The clinical data sheets were also adapted from a US version. Through close collaboration among the researchers in the participating sites, a common concept for each question in data sheets was established, emphasising similarities in the meanings of questions rather than exact linguistic equivalence. ${ }^{12}$ For most clinical diagnoses a definition was given in the data sheet. The intention of these procedures was to reduce bias by minimising differences in data collection and questionnaire design. 
Table 1 Demographic characteristics of patients with cataract in the four sites

\begin{tabular}{llllll}
\hline & $\begin{array}{l}\text { USA } \\
(n=766)\end{array}$ & $\begin{array}{l}\text { Denmark } \\
(n=291)\end{array}$ & $\begin{array}{l}\text { Manitoba } \\
(n=152)\end{array}$ & $\begin{array}{l}\text { Barcelona } \\
(n=200)\end{array}$ & $p$ Value \\
\hline Mean age (years) & 72.4 & 73.5 & 71.7 & 69.9 & $<0.01^{\star}$ \\
$\quad$ SD & 7.7 & 8.0 & 8.9 & 8.4 & \\
Female (\%) & 62.8 & 67.0 & 67.1 & 60.4 & $>0.05 \dagger$ \\
Living alone (\%) & 33.0 & 49.8 & 34.2 & 17.4 & $<0.01 \dagger$ \\
$\quad \begin{array}{l}\text { Working outside home (\%) } \\
\text { General health status }\end{array}$ & 18.9 & 19.0 & 21.1 & 7.7 & $<0.01 \dagger$ \\
$\quad$ SIP score $\$$ & 8.3 & 6.3 & 8.9 & 14.6 & $<0.01^{\star}$ \\
SD & 10.0 & 7.0 & 9.6 & 13.0 & \\
\hline
\end{tabular}

*ANOVA, $\mathrm{f}$ test.

$+\chi^{2}$ test.

$\ddagger$ SIP score ranges from 0 (indicating absence of dysfunction) to 100 (indicating the maximum level of dysfunction).

\section{STATISTICAL ANALYSIS}

For statistical analysis, a reduction in visual acuity to the level of finger counting was assigned a Snellen value of 0.02 ; a reduction to identification of hand movements a value of 0.01 ; and light perception a value of 0.005 . Mean visual acuity measures were calculated as the geometric mean based on $\log M A R$ values as suggested by Moseley and Jones. ${ }^{13}$ However, all visual acuity measures in the paper are transformed and presented as Snellen decimal fractions.

Tests for differences across sites were performed using $\chi^{2}$ tests for categorical variables and one way ANOVA for continuous variables. ${ }^{14}$ If differences were observed using ANOVA, modified $t$ tests corrected for multiple comparisons (Bonferroni method) were subsequently performed between all pairs of countries. ${ }^{14}$ The modified $t$ test is based on the pooled estimate of variance from all the groups, not just the pair being considered. The adjustment for multiple comparisons means that if $k$ paired comparisons are performed, the $\mathrm{p}$ value obtained from each test is multiplied by $k$.

A multiple linear regression model was fitted to examine whether preoperative visual acuity was associated with sociodemographic factors. As dependent variables we included age, sex, general health status (SIP score), working outside the home, and living alone. Dummy variables for sites were included. ${ }^{15}$

Table 2 Ocular comorbidity; clinical findings in eye scheduled for surgery (\%)

\begin{tabular}{|c|c|c|c|c|c|}
\hline & $\begin{array}{l}U S A \\
(n=766)\end{array}$ & $\begin{array}{l}\text { Denmark } \\
(n=291)\end{array}$ & $\begin{array}{l}\text { Manitoba } \\
(n=152)\end{array}$ & $\begin{array}{l}\text { Barcelona } \\
(n=200)\end{array}$ & $p$ Value ${ }^{\star}$ \\
\hline Hypermature cataract & 1.6 & 1.7 & 3.3 & 16.4 & $<0.001$ \\
\hline ARMD & 17.1 & 22.1 & 14.5 & 16.2 & $<0.001$ \\
\hline Retinal disorder & 6.9 & 3.1 & 5.9 & 18.1 & $<0.001$ \\
\hline Corneal decompensation & 1.4 & 4.8 & 3.9 & 2.5 & $<0.05$ \\
\hline Glaucoma & 9.3 & 4.1 & 10.5 & 3.6 & $<0.001$ \\
\hline \multicolumn{6}{|c|}{$\begin{array}{l}{ }^{\star} \chi^{2} \text { test. } \\
A R M D=\text { age related macular degeneration. }\end{array}$} \\
\hline \multicolumn{6}{|c|}{ Table 3 Surgical techniques applied (\%) } \\
\hline & $\begin{array}{l}U S A \\
(n=766)\end{array}$ & $\begin{array}{l}\text { Denmark } \\
(n=291)\end{array}$ & \multicolumn{2}{|c|}{$\begin{array}{l}\text { Manitoba } \\
(n=152)\end{array}$} & $\begin{array}{l}\text { Barcelona } \\
(n=200)\end{array}$ \\
\hline Phacoemulsification & 66.4 & 32.8 & \multicolumn{2}{|c|}{64.0} & 3.4 \\
\hline ECCE & 33.6 & 67.2 & \multicolumn{2}{|c|}{35.0} & 96.0 \\
\hline ICCE & 0.0 & 0.0 & \multicolumn{2}{|c|}{1.0} & 0.6 \\
\hline
\end{tabular}

$\chi^{2}$ test: $\mathrm{p}<0.001$

$\mathrm{ECCE}=$ standard extracapsular cataract extraction

ICCE $=$ intracapsular cataract extraction.

\section{Results}

Of 888 eligible patients initially referred for enrolment in the United States, $772(87 \%)$ eventually agreed to participate. For 766 $(99 \%)$ of these patients, both preoperative interview and clinical data were obtained. Of 311 eligible and enrolled Danish patients, 291 (93.6\%) eventually participated and had preoperative interview and clinical data. Of 226 eligible patients referred for enrolment in Manitoba, 159 (70.4\%) agreed to participate. For $152(95.6 \%)$ patients, both initial interview and clinical data were obtained. A total of 219 patients were referred for enrolment in Barcelona; 53\% were from the public sector and $47 \%$ from the private sector. After being contacted, 200 patients (91\%) agreed to participate, and interview and clinical data were obtained.

Basic demographic and social information for the four samples of patients with cataract is shown in Table 1 . The proportion of patients still working or volunteering outside home was larger in the United States (18.9\%), Manitoba $(21.1 \%)$, and Denmark $(19.0 \%)$ compared with Barcelona $(7.7 \%)$. A large variation was also seen in the proportion of patients living alone, ranging from $17.4 \%$ in Barcelona to $49.8 \%$ in Denmark.

The general health status (SIP score) varied significantly among sites, Barcelona having the poorest health status (Table 1). Also significantly different prevalence of ocular comorbidity were seen across all four sites (Table 2) $(\mathrm{p}<0.001, \mathrm{p}<0.05)$. Barcelona had a significantly higher prevalence of retinal disorders than the other three sites $(\mathrm{p}<0.001)$. Subanalysis showed that this was due to higher prevalence of proliferative diabetic retinopathy, myopic and lattice retinal degenerations. The types of surgical procedures performed are shown in Table 3 and indicates that the highest rates of phacoemulsification were seen in the United States and Manitoba.

Preoperative visual acuity was measured during the examination when the decision to perform cataract surgery was made (Table 4). No statistically significant difference was seen between mean visual acuity in the eye scheduled for surgery in Denmark (0.17) and in Manitoba (0.15) $\quad(p>0.05)$. A statistically significantly better visual acuity was seen in the United States (0.23) compared with the other sites $(p<0.001)$. A statistically lower mean visual acuity of 0.07 was seen in Barcelona compared with the other sites $(\mathrm{p}<0.001)$.

Including only eyes with no age related macular disease or retinal disorders $(n=919)$, the difference between mean preoperative visual acuity in the United States and Denmark was no longer significant (USA 0.27 versus Denmark 0.25, p>0.05) (Table 5). The mean preoperative visual acuity in the Manitoba sample (mean visual acuity 0.20 ) was significantly lower than in the United States and Danish samples $(p<0.001)$. Mean preoperative visual acuity in Barcelona (mean visual acuity 0.10 ) was significantly lower than that seen at any of the other site $(\mathrm{p}<0.001)$. 
Table 4 Best corrected preoperative visual acuity in the operative eye for the four samples (Snellen decimal fraction)

\begin{tabular}{|c|c|c|c|c|c|}
\hline & $U S A$ & Denmark & Manitoba & Barcelona & $p$ Value $^{*}$ \\
\hline \multicolumn{6}{|l|}{ Total sample: } \\
\hline No & 766 & 291 & 152 & 200 & \\
\hline Mean visual acuity & 0.23 & 0.17 & 0.15 & 0.07 & $<0.001$ \\
\hline \multicolumn{6}{|c|}{ Distribution of visual acuity: } \\
\hline$<0.05$ & $9 \%$ & $15 \%$ & $19 \%$ & $40 \%$ & \\
\hline$>0.05-\leqslant 0.10$ & $10 \%$ & $11 \%$ & $19 \%$ & $27 \%$ & \\
\hline$>0.10-\leqslant 0.25$ & $14 \%$ & $32 \%$ & $19 \%$ & $22 \%$ & \\
\hline$>0.25-\leqslant 0.40$ & $54 \%$ & $29 \%$ & $36 \%$ & $10 \%$ & \\
\hline$>0.40$ & $13 \%$ & $13 \%$ & $7 \%$ & $1 \%$ & \\
\hline \multicolumn{6}{|c|}{ Eyes with normal retina and macula only: } \\
\hline No & 554 & 169 & 102 & 94 & \\
\hline Mean visual acuity & 0.27 & 0.25 & 0.20 & 0.10 & $<0.001$ \\
\hline \multicolumn{6}{|c|}{ Distribution of visual acuity } \\
\hline$<0.05$ & $5 \%$ & $5 \%$ & $9 \%$ & $23 \%$ & \\
\hline$>0.05-\leqslant 0.10$ & $9 \%$ & $9 \%$ & $20 \%$ & $40 \%$ & \\
\hline$>0.10-\leqslant 0.25$ & $14 \%$ & $34 \%$ & $19 \%$ & $22 \%$ & \\
\hline$>0.25-\leqslant 0.40$ & $57 \%$ & $34 \%$ & $44 \%$ & $14 \%$ & \\
\hline$>0.40$ & $15 \%$ & $18 \%$ & $8 \%$ & $1 \%$ & \\
\hline
\end{tabular}

^ANOVA, f test.

Table 5 Preoperative VF-14 scores in the four samples (a score of 100 indicates no impairment, and of 0 indicates maximum impairment)

\begin{tabular}{lrrrrl}
\hline & USA & Denmark & Manitoba & Barcelona & p Value \\
\hline Total sample: & 766 & 291 & 152 & 200 & \\
No & 76 & 76 & 71 & 64 & $<0.001$ \\
Mean VF-14 score & 17 & 17 & 21 & 27 & \\
SD & 554 & 169 & 102 & 94 & \\
Eyes with normal retina and macula only: & 76 & 79 & 71 & 70 & $<0.001$ \\
No & 16 & 16 & 21 & 24 & \\
Mean VF-14 score & & & & & \\
SD & & &
\end{tabular}

^ANOVA, f test.

Table 6 Association between preoperative visual acuity and sociodemographic characteristics as shown in a linear regression model

\begin{tabular}{lclc}
\hline & $B$ & SE B & $p$ Value \\
\hline Constant & 0.80 & 0.13 & $<0.001$ \\
Dummy variables for site & & & \\
$\quad$ Site 1 & 0.18 & 0.04 & $<0.001$ \\
$\quad$ Site 2 & 0.13 & 0.03 & $<0.001$ \\
$\quad$ Site 3 & 0.52 & 0.04 & $<0.001$ \\
General health status & 0.003 & 0.001 & 0.01 \\
Age (years) & -0.003 & 0.002 & 0.09 \\
Working outside home (no/yes) & -0.02 & 0.03 & 0.52 \\
Living alone (no/yes) & 0.02 & 0.03 & 0.47 \\
Sex (male/female) & 0.004 & 0.026 & 0.88 \\
\hline
\end{tabular}

*SIP score, ranging from 0 (indicating absence of dysfunction) to 100 (indicating the maximum level of dysfunction).

When considering non-operative eyes (data not shown), no significant differences were seen between the United States (mean visual acuity 0.41), Denmark (mean visual acuity 0.43 ), and Manitoba (mean visual acuity 0.38 ) $(\mathrm{p}>0.05)$. However, a significantly lower mean visual acuity was seen in the Barcelona sample (mean visual acuity 0.20$)(\mathrm{p}<0.001)$.

Patients' self reported visual functional impairment was also compared across sites. Mean VF-14 scores were similar in the United States and Denmark ( $>0.05)$, but significantly lower (that is, more impairment) in Manitoba and Barcelona ( $\mathrm{p}<0.001)$ (Table 5). When considering eyes without age related macular disease or retinal disorders $(n=919)$, a similar pattern was observed (Table 5).

A regression model (Table 6) shows that preoperative visual acuity is significantly associated with general health status (SIP score) and marginally associated with age. However, the model also shows that the difference in preoperative visual acuity among sites is significant even after controlling for these variables.

Variation was observed in duration of the patients' waiting time from the examination was done and until surgery was actually performed. The mean time was less than 3 months in the United States, 3.3 months in Barcelona, 5.4 months in Manitoba, and 1 month in Denmark.

\section{Discussion}

A comparison of preoperative clinical status of patients with cataract across four international sites has shown differences in both visual acuity and VF-14 measures. Including all categories of patients, preoperative visual acuity of the operated eye was significantly better in the United States compared with the other three sites. The worst mean visual acuity was observed in Barcelona. VF-14 scores followed a similar gradient.

Within the North America, the mean visual acuity score and the mean VF-14 score were significantly better in the United States than the mean score in Manitoba even though the two samples of patients appear to be similar with regard to demographic characteristics and prevalence of ocular comorbidity. The variation still existed when restricting the sample to eyes with healthy retina and macula. The cause of this variation is probably multifactorial. The observed difference in preoperative visual status may in part be due to reliance on more restrictive indications for surgery in Manitoba. Another contributing factor may be a relatively lower surgical capacity leading to a problem of access to care in the Manitoba healthcare system compared with the United States. A third factor may be that a larger proportion of US patients request surgery at a somewhat lower level of visual impairment. In the present study, the weight of these different factors could not be determined.

Although differences were found in comparisons between the United States and Denmark based on all categories of patients, no statistically nor clinically significant difference was found in preoperative visual acuity and VF-14 scores, when restricting the samples to patients with normal retina and macula. The two samples also appear to be similar with regard to ocular comorbidity. The findings suggest that indications for surgery are similar in the United States and Denmark and more liberal than in Manitoba and Barcelona.

The mean preoperative visual acuity and VF-14 score was statistically as well as clinically significantly lower in Barcelona compared with any other site. The lower mean visual acuity and VF14 scores in Barcelona are consistent with the higher prevalence of hypermature cataract observed in the Barcelona sample. Even after restricting the sample to patients with no retinal or macular disorders, the difference in visual status between Barcelona and the other sites remained. This suggests that the difference in visual status is not likely to be due to differences in the ocular case mix of the samples. As noted in compari- 
sons between the United States and Manitoba, differences in preoperative visual status might be due to cultural differences in the patient demand for visual functional capacity and in perceptions of how far a disease should progress before professional guidance is obtained, and/or it could represent an access problem in Barcelona.

Although visual acuity is a simple indicator of visual status, it is also widely accepted that the cataract surgeon should be guided as much by the visual impairment reported by the patient as by the surgeon's own clinical findings. ${ }^{16}$ It has also been shown that overall visual function in daily life and visual acuity are only moderately correlated. ${ }^{4}$ Therefore, both visual acuity and a measure of functional impairment should be used when comparing indications for cataract surgery across sites. The validity of our findings are strengthened by the observation of a similar pattern of results for both visual acuity and VF-14 scores.

In the present study, indications for surgery as measured by preoperative visual acuity was significantly associated with general health status of the patients and marginally with age. This is in accordance with previous studies showing that the decision to perform cataract surgery is significantly associated with demographic and lifestyle factors such as age, sex, race, income, ${ }^{17}$ and being employed. ${ }^{18}$ However, in the present study these factors alone could not explain the variation in preoperative visual acuity which points to other explanatory factors such as access to care, practice style, and patient demand as discussed above.

The recommendations for surgery might potentially be adjusted to the anticipated length of time a patient has to be on a waiting list for surgery. A long waiting time could introduce a clinical routine of scheduling surgery before it is actually needed, because the cataract supposedly progress during the waiting time. However, we believe that the mean waiting time at all four sites was so short relative to the natural history of the disease that this would not be a factor in explaining differences in preoperative clinical status.

Some limitations of this study must be acknowledged. Lack of representativeness of the samples might affect the appropriateness of the conclusions. Although consecutive samples were drawn from multiple clinical practices at each site, there could potentially be selection bias in the sampling strategy. The facilities from which the samples were collected might not represent the healthcare systems of the study sites. To avoid such bias in the inclusion of facilities, we invited all cataract surgeons in Manitoba, all cataract surgeons in the public sector in Denmark, and random samples of cataract surgeons in Barcelona and the United States to participate. However, in the United States, the sampling was more rigorous. Three different metropolitan areas had to be selected as the coordination of the study would have been much more complicated if participants had been enrolled from all areas of the United States.
Also the sampling of patients at each facility could potentially be biased, although unequivocal and simple criteria for inclusion were used. In the United States and Denmark, however, medical charts have been reviewed for eligible patients who declined to participate and no differences from the actual sample were found..$^{19}{ }^{20}$ We believe that the samples are representative of the healthcare systems in these sites. A review of non-participants in Manitoba and Barcelona was not possible in the present study.

To avoid bias during data collection, great care was taken to ensure that interviews and data sheets were similar across sites. Definitions were given in the data sheets for most clinical diagnoses and tend towards minimising bias due to differences in local definition of medical conditions.

\section{Conclusion}

In the present study, we have observed significant differences in threshold or indications for cataract surgery in the four sites. Some of the variation could be explained by differences in sociodemographic characteristics. However, contributing factors might be access to care, practice style, and patient demand.

1 Bernth-Petersen P, Bach E. Epidemiologic aspects of cataract surgery. II Regional variation in frequencies. Acta Ophthalmol 1983;61:397-405.

2 Desai P. The National Cataract Survey III. Process features. Eye 1993;7:667-71.

3 Javitt JC, Kendix M, Tielsch JM, et al. Geographic variation in utilization of cataract surgery. Med Care 1995;33:90105.

4 Steinberg EP, Tielsch JM, Schein OD, et al. The VF14. An index of functional impairment in patients with cataract. Arch Ophthalmol 1994;112:630-8.

5 Sigmund H, Danneskiold-Samsoe B. Cataract surgery at hospital and in private practice. Danish Hospital Institute, report 92.01. Copenhagen 1992 (in Danish).

6 Cassard SD, Patrick DL, Damiano AM, et al. Reproducibility and responsiveness of the VF-14. An index of functional impairment in patients with cataract. Arch Ophthalmol 1995;113:1508-13.

7 Alonso J, Espallargues M, Andersen TF, et al. International applicability of the VF-14, an index of visual function in patients with cataract. Ophthalmology 1997;104:799-807.

8 Bergner M, Bobbit RA, Carter WB, et al. The Sickness Impact Profile: development and final revision of a health status measure. Med Care 1981;19:787-805.

9 de Bruin AF, Witte LP, Stevens F, et al. Sickness Impact Profile: the state of art of a generic functional status measure. Soc Sci Med 1992;35:1003-14.

10 Brislin RW, Lonner WJ, Thorndike RM. Cross-national research methods. New York: John Wiley, 1986:32-81.

11 Badia X, Alonso J. Validity and reproducibility of Spanish Badia X, Alonso J. Validity and reproducibility of Spanish
version of Sickness Impact Profile. I Clin Epidemiol 1995;49:359-65.

12 Anderson GF, Alonso J, Kohn LT, et al. Analyzing health outcomes through international comparisons. Med Care 1994;32:526-34

13 Moseley MJ, Jones HS. Visual acuity: calculating appropriate averages. Acta Ophthalmol 1993;71:296-300.

4 Altman DG. Practical statistics for medical research. London: Chapman and Hall, 1991:205-15.

15 Altman DG. Practical statistics for medical research. London: Chapman and Hall, 1991:339.

16 Clinical Practice Guidelines Panel. Cataract in adults: management of functional impairment. Rockville, MD: Agency for Health Care Policy and Research Pub No 93-0542, February 1993.

17 Escarce JJ. Would eliminating differences in physician practice style reduce geographic variation in cataract surgery tice style reduce geographic variation
rates? Med Care 1993;31:1106-18.

18 Keefe JE, McCarty CA, Chan WP, et al. Relative importance of VA, patient concern patient lifestyleon referral fo cataract surgery. Invest Ophthalmol Vis Sci 1996;37:S183.

19 Schein OD, Steinberg EP, Javitt JC, et al. Variation in cataract surgery practice and clinical outcomes. Ophthalmology 1994;101:1142-52.

20 Norregaard JC, Bernth-Petersen P, Andersen TF. Visual impairment and general health among Danish cataract patients. Acta Ophthalmol 1996;74:598-603. 